\title{
Erratum: Marginal Gap Evaluation of Metal Onlays and Resin Nanoceramic Computer-Aided Design and Computer-Aided Manufacturing Blocks Onlays
}

Nor Faharina Abdul Hamid ${ }^{1}$ Wan Zaripah Wan Bakar ${ }^{2}$ Zaihan Ariffin ${ }^{2}$

${ }^{1}$ Restorative Dentistry Unit, Faculty of Dentistry, Universiti

Teknologi MARA, Sungai Buloh Campus, Jalan Hospital, Selangor, Malaysia

${ }^{2}$ Conservative and Prosthodontics Unit, School of Dental Sciences Health Campus, Universiti Sains Malaysia, Kubang Kerian, Kota

Bahru, Kelantan, Malaysia

Eur J Dent 2020;13:e1
Address for correspondence Wan Zaripah Wan Bakar, BDS, GDCD, DCD, Conservative and Prosthodontics Unit, School of Dental Sciences Health Campus, Universiti Sains Malaysia,16150, Kubang Kerian, Kota Bahru, Kelantan, Malaysia (e-mail: wzaripah@usm.my).

ERRATUM 